\title{
Assessment of mHealth Interventions: Need for New Studies, Methods, and Guidelines for Study Designs
}

Roxana Ologeanu-Taddei, PhD

TBS Business School, Toulouse, France

Corresponding Author:

Roxana Ologeanu-Taddei, $\mathrm{PhD}$

TBS Business School

1, place Alphonse Jourdain

Toulouse, 31068

France

Phone: 33561294851

Email: r.ologeanu-taddei@tbs-education.fr

\section{Abstract}

This viewpoint argues that the clinical effects of mobile health (mHealth) interventions depends on the acceptance and adoption of these interventions and their mediators, such as usability of the mHealth software, software performance and features, training and motivation of patients and health care professionals to participate in the experience, or characteristics of the intervention (eg, personalized feedback).

(JMIR Med Inform 2020;8(11):e21874) doi: 10.2196/21874

\section{KEYWORDS}

eHealth; mHealth; usability; management; survey; trust; guidelines; evaluation

\section{Background}

In the past years, designs of research studies in medical journals have been formalized according to the reporting guidelines of academic associations, international consortia, and publishers, which enable publications of clinical trials, systematic reviews, and meta-analyses. The use of mobile health (mHealth) technologies is expected to increase, creating new paths for health care delivery. However, there are no specific guidelines to enable researchers to design and present their studies and results on this topic, except for the existing guidelines on randomized clinical trials (RCTs), which can be used for certain assessments of mHealth interventions.

\section{Measures of Clinical Effects Require Opening the Black Box of Information Technology}

There is no doubt that RCTs are useful to assess the clinical outcomes and effectiveness of mHealth. In this context, regulatory bodies such as the US Food and Drug Administration (FDA) [1] and European regulations have recently updated the requirements for clinical proofs of mHealth solutions. Nevertheless, the focus on RCT methods leads to the black boxing of mHealth interventions [2], which means that the technology is considered as a homogeneous device or as a pharmaceutical substance. This view misses the main characteristic of an mHealth intervention (and overall, that of information technology [IT]) — the embeddedness of data and clinical processes (as reflected in the guidelines for diagnosis and personalized monitoring). Therefore, assessing the clinical effect of an mHealth intervention should disentangle the effect of a new process of personalized monitoring, the effect of the ubiquitous access enabled by mobile devices, and the comprehension and adoption of clinical guidelines implemented into the application. In addition, mHealth solutions may differ from one another because of their different designs of these processes.

\section{Need of Standard Guidelines to Assess Technology and Mediators of Outcomes}

Moreover, the clinical effect of mHealth solutions depends on the acceptance and adoption of these solutions and their mediators, such as usability of the mHealth software, software performance and features, training and motivation of patients and health care professionals to participate in the experience, or characteristics of the intervention (eg, personalized feedback). For example, a clinical effect such as survival benefits for patients with cancer who use a surveillance mHealth app depends on the acceptance and adoption of the app, which can 
be influenced by the usability of the app and patients' prior experience in using mobile apps, motivation or trust in IT, or other alternative mediators contributing to the main reported outcome; this influences are often neglected by RCTs.

Settings of mHealth interventions must be carefully described and assessed in hypothesis-generating studies [3], such as observational studies and case reports. These studies can identify specific moderators and mediators that state for whom and under what conditions the health intervention works [3]. Moderators may identify population groups with possible causal mechanisms or courses of illness. The mHealth mediators identify possible causal mechanisms, meaning causal links between the intervention and the outcome, through which the intervention may achieve its effects [3]. As a next step, these moderators and mediators should be considered as stratification variables in forthcoming RCTs focused on hypothesis testing. Otherwise, RCTs are likely to be based on weak assumptions rather than empirical evidence.

\section{Beyond Effectiveness: Risk Assessment}

In addition, RCTs need to be complemented by other clinical trials and case reports to assess safety risks [4] and unintended consequences of mHealth. The acknowledgment of these risks is at the core of the updated regulations for medical devices, which include software and mHealth. However, in most cases, assessments of mHealth safety risks are conducted separately [5], for example, in feasibility or usability studies, which use different methods of varying rigor and do not generate cumulative knowledge. In addition, case reports on the adoption stages of mHealth solutions should be inspired by engineering methods (eg, fault tree analysis rather than pharmacovigilance studies). Moreover, relevant and cumulative knowledge can be gathered if publications on the issues of usability and user acceptance are presented not only in health informatics journals but also in major medical journals, as these issues cause clinical effects. For example, the Journal of the American Medical Association (JAMA) Network advises authors to use the guidelines of the EQUATOR (Enhancing the QUAlity and Transparency Of health Research) network [6], which include guidelines related to "economic studies." Similar initiatives should be undertaken for studies concerning mHealth. In recent years, cumulative knowledge has been gathered on the risks associated with poor usability of health IT [7]. In line with this literature, a step forward was taken only for mHealth solutions, which are qualified as medical devices [8]. However, even for those applications, national and international regulations (ie, CE marking in Europe or FDA regulations) and harmonized standards (ie, EN 62366 advised for CE marking in Europe) strengthened the requirements for premarket certifications but did not standardize a threshold for usability or technical performance. We must recognize that recommending the minimum required sample size (eg, 15 users identified by user profile numbers) makes an improvement to the summative usability assessment method [9]. It is also necessary to assess user profiles accurately. These user profiles may be related to health care occupations (eg, clinical secretaries, physicians, or nurses), different health departments (eg, infectious diseases or pediatrics), and social or demographic variables. In addition, several other characteristics (eg, computer literacy, prior experience of using mobile apps, and users' engagement or trust in IT) may mediate or moderate mHealth effects and therefore could be taken into account to refine user profiles. Further research is needed on these moderators and mediators to use them as criteria for construction of user profiles. Although such research can be complex and costly, it is relevant and useful.

\section{Need of Studies in Implementation and Adoption Stages}

The implementation of mHealth solutions (beyond pilots) in the market and their user adoption stages introduce new contextual and technological factors, such as low technical performance, lack of interoperability with existing systems, or misfit with existing clinical practices. Pilot studies often benefit from specific resources - both financial and human-and from high motivation of the patients and health care professionals involved. These factors may be missing in the latter stages of implementation and adoption, influencing the outcome achievement or introducing risks to patient safety. Although these challenges cannot be experimentally controlled, they may be assessed cautiously in rigorous qualitative and statistical studies. In addition, the moderators and mediators of mHealth interventions, such as engagement levels [10], should be investigated more systematically.

We have to mention that the EQUATOR network published guidelines for the reporting of mobile phone-based health interventions [11]. Formed by the World Health Organization's panel of experts, these guidelines are useful to improve the transparency and harmonization of the reporting of mHealth, enabling comparisons and meta-reviews. These criteria include usability/content testing and user feedback. Nevertheless, a new step must be taken toward formulating guidelines on study designs and methods of the assessment of user feedback on mHealth interventions.

\section{Moving Forward: Formal Guidelines for Study Designs on Real-World Usage}

The evaluation of moderators and mediators in pilots should be followed by larger surveys and follow-ups during the whole life cycle of the mHealth technology [5]. The protocols of these studies should be inspired by the rigor of protocols of clinical investigations while considering the relevant factors specific to mHealth. Open trials, observational studies, and case reports should be conducted to measure mediators beyond a specific clinical setting (the variables and low sample size of which could introduce serious bias). In addition, anecdotal reports and qualitative studies should use common frameworks, which will facilitate systematic reviews and afford transferability. The knowledge generated can thus inform policy decisions.

Moreover, anecdotal reports of suspected adverse reactions related to the use of mHealth (along with reports of health IT-related adverse events) should be encouraged and published in medical journals, as mHealth can induce specific errors related to the use of technology. These issues have been emphasized as crucial for more than 20 years, during which 
numerous studies have shown that bad informatics can have fatal consequences [5]. If the new European regulations on medical devices (which include mHealth solutions) require real-life, postmarket, clinical, and risk assessment follow-ups of these devices, new methods and frameworks should be elaborated with scientific rigor and inspired by different academic disciplines, such as engineering and social sciences. Building on the guidelines for research presentations and knowledge from medical informatics and risk engineering [12], it is time to make rigorous evaluations and formalize guidelines for research presentations, enabling evidence-based mHealth interventions.

\section{Conflicts of Interest}

None declared.

\section{References}

1. US Food and Drug Administration. Policy for Device Software Functions and Mobile Medical Applications: Guidance for Industry and Food and Drug Administration staff. 2019. URL: https://www.fda.gov/media/80958/download [accessed 2020-08-15]

2. Free C, Phillips G, Watson L, Galli L, Felix L, Edwards P, et al. The effectiveness of mobile-health technologies to improve health care service delivery processes: a systematic review and meta-analysis. PLoS Med 2013;10(1):e1001363 [FREE Full text] [doi: 10.1371/journal.pmed.1001363] [Medline: 23458994]

3. Kraemer HC, Wilson GT, Fairburn CG, Agras WS. Mediators and moderators of treatment effects in randomized clinical trials. Arch Gen Psychiatry 2002 Oct;59(10):877-883. [doi: 10.1001/archpsyc.59.10.877] [Medline: 12365874]

4. Lewis TL, Wyatt JC. mHealth and mobile medical Apps: a framework to assess risk and promote safer use. J Med Internet Res 2014 Sep 15;16(9):e210 [FREE Full text] [doi: 10.2196/jmir.3133] [Medline: 25223398]

5. Ammenwerth E, Shaw NT. Bad health informatics can kill--is evaluation the answer? Methods Inf Med 2005;44(1):1-3. [Medline: 15778787]

6. EQUATOR Network. URL: https://www.equator-network.org/ [accessed 2020-08-15]

7. Zapata BC, Fernández-Alemán JL, Idri A, Toval A. Empirical studies on usability of mHealth apps: a systematic literature review. J Med Syst 2015 Feb;39(2):1. [doi: 10.1007/s10916-014-0182-2] [Medline: 25600193]

8. Keutzer L, Simonsson US. Medical Device Apps: An Introduction to Regulatory Affairs for Developers. JMIR Mhealth Uhealth 2020 Jun 26;8(6):e17567 [FREE Full text] [doi: 10.2196/17567] [Medline: $\underline{32589154]}$

9. Borsci S, Londei A, Federici S. The Bootstrap Discovery Behaviour (BDB): a new outlook on usability evaluation. Cogn Process 2011 Feb;12(1):23-31. [doi: 10.1007/s10339-010-0376-6] [Medline: 21046191]

10. Yang Q, Van Stee SK. The Comparative Effectiveness of Mobile Phone Interventions in Improving Health Outcomes: Meta-Analytic Review. JMIR Mhealth Uhealth 2019 Apr 03;7(4):e11244 [FREE Full text] [doi: 10.2196/11244] [Medline: 30942695]

11. Agarwal S, LeFevre AE, Lee J, L'Engle K, Mehl G, Sinha C, WHO mHealth Technical Evidence Review Group. Guidelines for reporting of health interventions using mobile phones: mobile health (mHealth) evidence reporting and assessment (mERA) checklist. BMJ 2016 Mar 17;352:i1174. [doi: 10.1136/bmj.i1174] [Medline: 26988021]

12. Jones R, Mateer J. Indirect risk related failures of Medical Information Systems. 2020 Aug 15 Presented at: 14th IEEE Conference on Industrial Electronics and Applications (ICIEA). IEEE; 2019; Xi'an, China p. 994-999. [doi: 10.1109/iciea.2019.8834057]

\section{Abbreviations}

EQUATOR: Enhancing the QUAlity and Transparency Of health Research

FDA: Food and Drug Administration

IT: informational technology

JAMA: Journal of the American Medical Association

mHealth: mobile health

RCT: randomized clinical trial 
Edited by C Lovis; submitted 27.06.20; peer-reviewed by $R$ Marcilly, $Q$ Yang; comments to author 03.08.20; revised version received 05.09.20; accepted 12.09.20; published 18.11.20

Please cite as:

Ologeanu-Taddei $R$

Assessment of mHealth Interventions: Need for New Studies, Methods, and Guidelines for Study Designs

JMIR Med Inform 2020;8(11):e21874

URL: http://medinform.jmir.org/2020/11/e21874/

doi: $10.2196 / 21874$

PMID: 33206060

CRoxana Ologeanu-Taddei. Originally published in JMIR Medical Informatics (http://medinform.jmir.org), 18.11.2020. This is an open-access article distributed under the terms of the Creative Commons Attribution License (https://creativecommons.org/licenses/by/4.0/), which permits unrestricted use, distribution, and reproduction in any medium, provided the original work, first published in JMIR Medical Informatics, is properly cited. The complete bibliographic information, a link to the original publication on http://medinform.jmir.org/, as well as this copyright and license information must be included. 